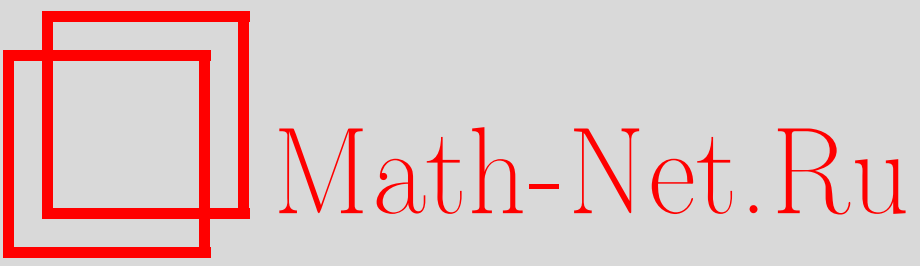

И. Г. Корепанов, С. Сайто, Конечномерные аналоги струнной $s \leftrightarrow t$ дуальности и уравнение пентагона, ТМФ, 1999, том 120, номер 1, 54-63

DOI: https://doi.org/10.4213/tmf759

Использование Общероссийского математического портала Math-Net.Ru подразумевает, что вы прочитали и согласны с пользовательским соглашением

http://www . mathnet.ru/rus/agreement

Параметры загрузки:

IP : 3.82 .47 .9

26 апреля 2023 г., 18:19:50 
ТЕОРЕТИЧЕСКАЯ

И МАТЕМАТИЧЕСКАЯ

ФИЗИКА

Том 120, № 1

июль, 1999

(C) 1999 г.

И. Г. Корепанов* , С. Сайто ${ }^{\dagger}$

\section{КОНЕЧНОМЕРНЫЕ АНАЛОГИ СТРУННОЙ $s \leftrightarrow t$ ДУАЛЬНОСТИ И УРАВНЕНИЕ ПЕНТАГОНА}

Одна из форм функционального уравнения пентагона (ФУП), встречающегося в теории интегрируемых моделей, предложена для алгебраического описания явления, известного в физике как $s \leftrightarrow t$ дуальность. Приведены два простых геометрических при-

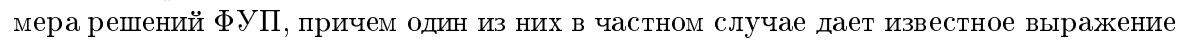

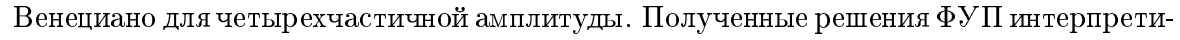
руются в терминах соотношений в группах Ли.

\section{1. ВВЕДЕНИЕ}

Одним из наиболее характерных свойств рассеяния в теории струн, а также в некоторых процессах сильного взаимодействия является так называемая $s \leftrightarrow t$ дуальность. Она может быть изображена диаграммой, приведенной на рис. 1.

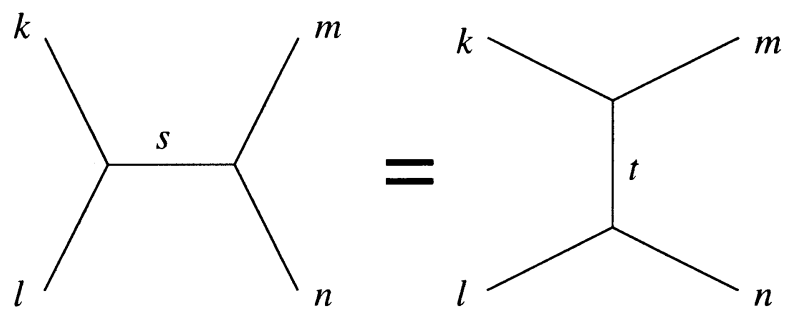

Рис. 1. Уравнение $s \leftrightarrow t$ дуальности.

Мы принимаем здесь точку зрения, что четырехточечные амплитуды $A(k, l, m, n)$, где $k, l, m, n$-некоторые "квантовые числа", получаются в обеих частях рис. 1 из трехточечных амплитуд $A(k, l, m)$ интегрированием по квантовому числу, отвечающему внут-

* Южно-Уральский государственный университет, Челябинск, Россия.

E-mail: igor@prima.tu-chel.ac.ru

† Токийский городской университет, Токио, Япония. E-mail: saito@phys.metro-u.ac.jp 


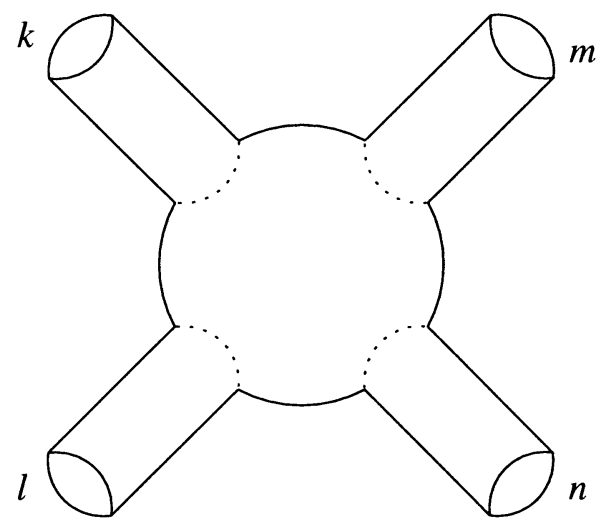

Рис. 2. Диаграмма, "топологически" объясняющая $s \leftrightarrow t$ дуальность.

ренней линии, так что аналитически дуальность выглядит так:

$$
\int A(k, l, s) A(s, m, n) d \mu(s)=\int A(k, m, t) A(t, l, n) d \mu(t),
$$

где $\boldsymbol{\mu}$ - некоторая мера. В формуле (1) трехточечная амплитуда считается симметричной по всем своим аргументам. Если это не так, то формулу (1) следует уточнить, как мы увидим ниже в разделе 4 .

Как известно, вследствие $s \leftrightarrow t$ дуальности резко уменьшается количество фейнмановских диаграмм: любые две диаграммы с одинаковым количеством внешних линий и циклов эквивалентны.

Какие математические структуры составляют основу такой дуальности? Конечно, в рамках обычной теории струн ее можно объяснить “геометрически", сказав, что обеим частям диаграммы на рис. 1 соответствует на самом деле одна и та же "струнная диаграмма", или мировой лист струны (рис. 2).

Предположим, однако, что мы хотим найти обший алгебраический механизм $s \leftrightarrow t$ дуальности, не зависящий от таких диаграмм и дающий надежду на новые возможности построения теорий, похожих на теорию струн.

Теория струн тесно связана со многими областями математики. Нас больше всего интересуют ее связи с интегрируемыми моделями. Поэтому мы отметим здесь соответствие между струнами и солитонами [1-3] и то, что струнные амплитуды удовлетворяют уравнению Янга-Бакстера [4].

Сушествуют, однако, разные виды фундаментальных уравнений, ответственных за интегрируемость. Наиболее важным считается уравнение тетраэдров (УТ), связанное c $(2+1)$-мерной интегрируемостью: квантовое УТ [5] для квантовых моделей и функциональное УТ $[6,7]$ как для классических, так и для квантовых моделей. В то же время различные уравнения неразрывно связаны друг с другом. В частности, УТ связано с уравнением пентагона [8]. 


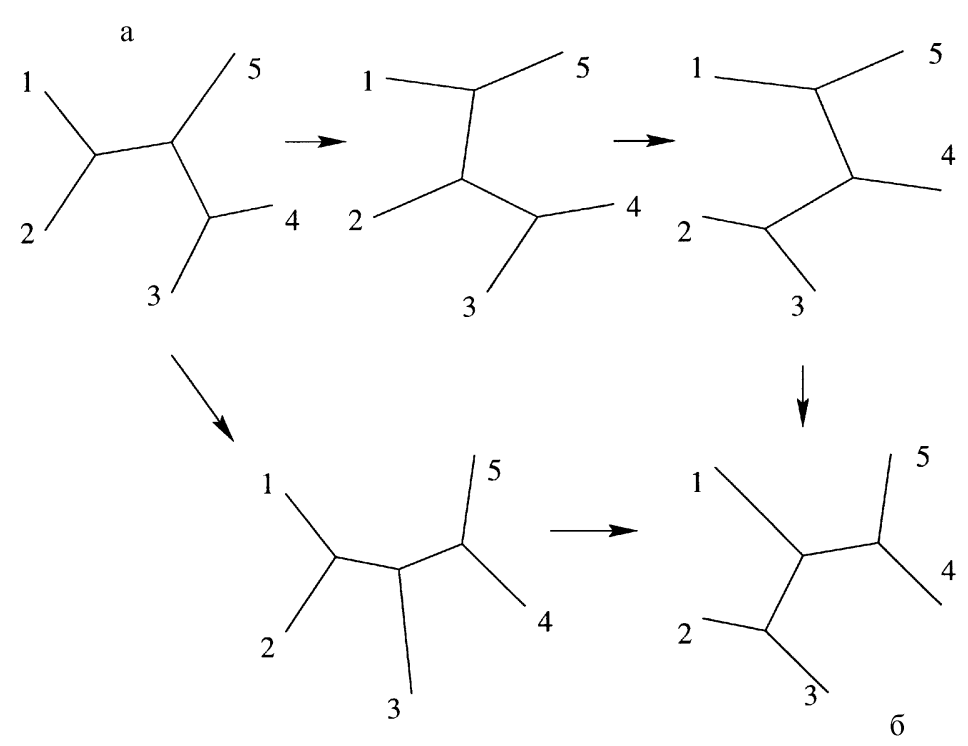

Рис. 3. Схема, поясняющая два способа преобразования квантовых чисел.

В этой статье мы приводим аргументы в пользу того, что математической структурой, ответственной за $s \leftrightarrow t$ дуальность, является функциональное уравнение пентагона (ФУП). Мы объясняем это на простых примерах. Как известно, теория струн тесно связана с бесконечномерными группами. Тем не менее мы считаем естественным начать $c$

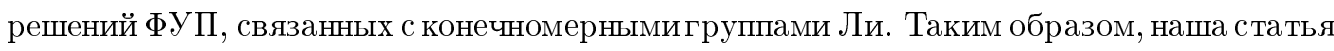
имеет своей скромной целью показать, что может сушествовать алгебраический меха-

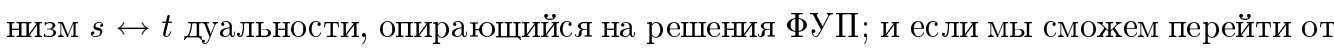
конечномерных к бесконечномерным группам (что кажется весьма вероятным), мы получим широкий класс новых теорий типа теории струн.

Ниже в разделе 2 мы объясняем, что такое ФУП и как оно естественно возникает при рассмотрении $s \leftrightarrow t$ дуальности. В разделах 3 и 4 приводятся две простые геометрические конструкции трехточечных амплитуд, обладаюших свойством дуальности. Амплитуда из раздела 4 обобшает известную четырехчастичную амплитуду Венециано. Наконец, в разделе 5 показано, что наши конструкции могут быть описаны алгебраически в терминах соотношений в группах Ли, а именно в группе движений евклидовой плоскости и группе Гейзенберга.

\section{2. ФУНКЦИОНАЛЬНОЕ УРАВНЕНИЕ ПЕНТАГОНА}

Наша идея механизма дуальности очень проста. Предположим, что для любых фиксированных квантовых чисел на внешних линиях обеих частей диаграммы на рис. 1 существует некий закон соответствия $f: t \mapsto s$ такой, что

$$
t=f(s) \Rightarrow A(k, l, s) A(s, m, n) d \mu(s)=A(k, m, t) A(t, l, n) d \mu(t) .
$$




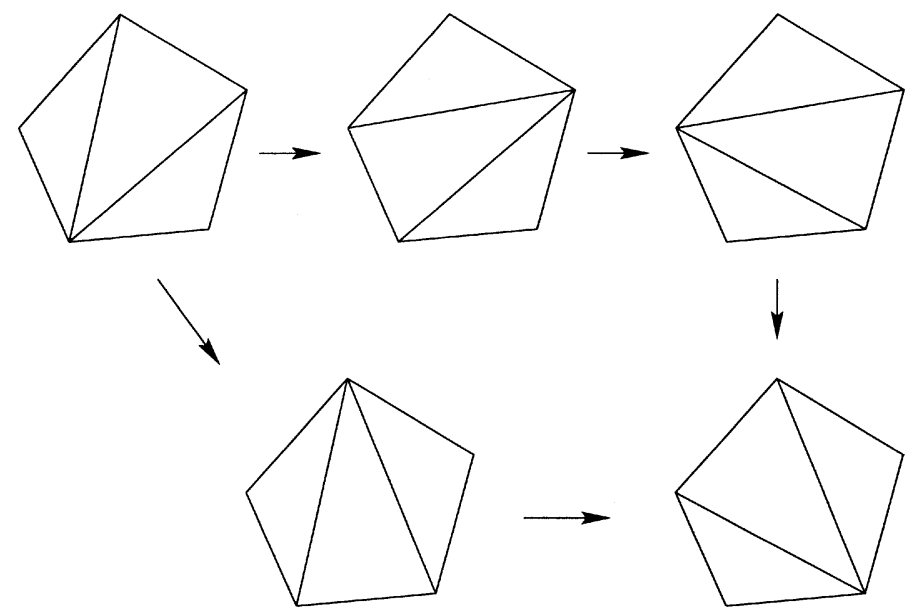

Рис. 4. Уравнение пентагона.

Здесь подразумевается, что функция $f$ зависит также от "внешних" переменных $k, l$, $m$ и $n$.

Ясно, что условия (2) достаточно для выполнения свойства (1). Рассмотрим теперь диаграмму рассеяния "а" на рис. 3 и преобразуем ее в диаграмму "б” двумя способами, как показано на рис. 3. Предположим, что всем линиям диаграммы "а" (включая внутренние) были сопоставлены некоторые квантовые числа. Применяя функцию $f$, мы получаем квантовые числа и для диаграммы “б”. Весьма естественно потребовать, чтобы функция $f$ удовлетворяла условию совместности: две последовательности преобразований на рис. 3 должни приводить к одинаковым квантовылм числам на диаграмме "б". А это условие совместности есть не что иное, как одна из разновидностей ФУП.

На рис. 4 изображен пентагон (пятиугольник), “двойственный по Пуанкаре” диаграмме рис. 3. При этом вершины диаграммы рис. 3 представляются треугольниками, а преобразованию диаграммы рис. 1 соответствуют удаление диагонали четырехугольника и замена ее другой диагональю.

Отметим, что переменные (квантовые числа) сопоставлены ребрам (сторонам и диагоналям) пентагона. Существуют и другие разновидности ФУП, в которых переменные принадлежат, например, самим треугольникам, см. [8, 9].

\section{3. ГЕОМЕТРИЧЕСКАЯ ДУАЛЬНОСТЬ ДЛЯ ДЛИН РЕБЕР}

Геометрическая картинка рис. 4 сразу подсказывает возможный выбор функции $f$. А именно, изобразим (рис. 5) “двойственную по Пуанкаре” конфигурацию к диаграмме рис. 1 на евклидовой плоскости и возьмем длинь ребер в качестве "квантовых чисел". 


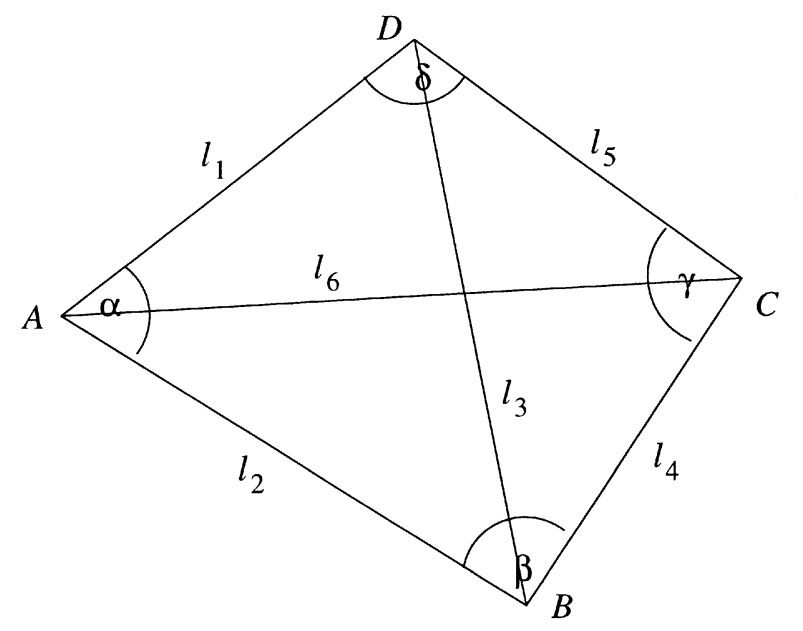

Рис. 5. Длины ребер как квантовые числа.

Если длины ребер $l_{1}, l_{2}, l_{3}, l_{4}$ и $l_{5}$ на рис. 5 заданы, то $l_{6}$ определяется из уравнения

$$
S_{A B D}+S_{B C D}=S_{A B C}+S_{A C D}
$$

где $S \ldots$ - плошадь соответствуюшего треугольника, выраженная через длины его сторон. Например,

$$
S_{A B D}=S\left(l_{1}, l_{2}, l_{3}\right)=\frac{1}{4} \sqrt{\left(l_{1}+l_{2}+l_{3}\right)\left(l_{2}+l_{3}-l_{1}\right)\left(l_{3}+l_{1}-l_{2}\right)\left(l_{1}+l_{2}-l_{3}\right)} .
$$

То, что такое преобразование $f: l_{3} \mapsto l_{6}$ удовлетворяет уравнению пентагона, очевидно из геометрических соображений (пятиугольник, длины всех сторон и двух диагоналей которого заданы, является “твердым телом", в котором расстояние между любыми двумя точками фиксировано и не зависит от цепочки алгебраических преобразований, посредством которых мы его вычислили).

Рассмотрим очевидное соотношение

$$
d(\alpha+\gamma)=-d(\beta+\delta)
$$

для углов на рис. 5 , и пусть стороны четырехугольника $A B C D$ заданы и только его диагонали могут меняться. Используя формулы типа

$$
\sin \alpha=\frac{2 S_{A B D}}{l_{1} l_{2}}, \quad \cos \alpha=\frac{l_{1}^{2}+l_{2}^{2}-l_{3}^{2}}{2 l_{1} l_{2}}
$$

мы найдем

$$
d \alpha=-\frac{d \cos \alpha}{\sin \alpha}=\frac{l_{3} d l_{3}}{2 S_{A B D}}
$$




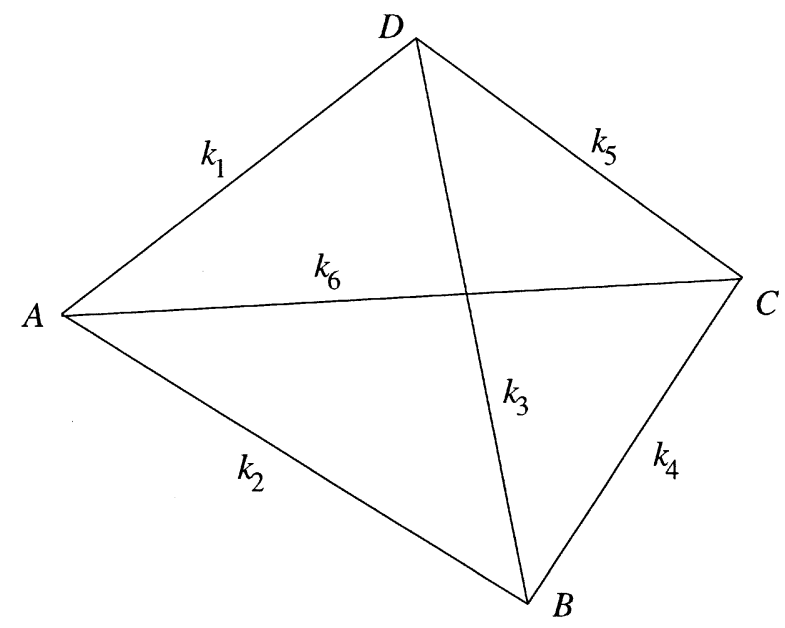

Рис. 6. Угловые коэффициенты как квантовые числа.

и аналогично для $\beta, \gamma$ и $\delta$. Подставляя эти дифференциалы углов в (4) и принимая во внимание уравнение (3), нетрудно вывести соотношение

$$
\frac{l_{3} d l_{3}}{S_{A B D} \cdot S_{B C D}}=-\frac{l_{6} d l_{6}}{S_{A B C} \cdot S_{A C D}} .
$$

Соотношение (5) вместе с (3) подсказывает следующий вид трехточечной амплитуды $A\left(l_{1}, l_{2}, l_{3}\right)$ и меры $d \mu(l)$, удовлетворяющих условию $(2)$ :

$$
A\left(l_{1}, l_{2}, l_{3}\right)=\frac{e^{\lambda S\left(l_{1}, l_{2}, l_{3}\right)}}{S\left(l_{1}, l_{2}, l_{3}\right)},
$$

где $\lambda$ - произвольная фиксированная константа, и

$$
d \mu(l)=l d l .
$$

Что касается знака минус в (5), его роль становится ясной при выборе пути интегрирования по $l_{3}$ и/или $l_{6}$. Здесь, как представляется, есть некоторая свобода. Если рассматривать длины как комплексные переменные, уравнение (3) при заданных $l_{1}, l_{2}$, $l_{4}$ и $l_{5}$ определяет некоторую риманову поверхность, точки которой суть пары $\left(l_{3}, l_{6}\right)$. Поэтому, возможно, в качестве контуров интегрирования можно взять какие-то циклы на этой поверхности. Здесь мы не будем рассматривать такую возможность, а просто наивно примем, что $A B C D$ - выпуклый четырехугольник на обычной вещественной евклидовой плоскости, и пусть $l_{3}$ изменяется от своего наименьшего значения, совместимого с этим условием (и с данными $l_{1}, l_{2}, l_{4}$ и $l_{5}$ ), до своего наибольшего значения. Тогда ясно, что $l_{6}$ изменяется от своего наибольшего до своего наименьшего значения. При изменении направления интегрирования по $l_{6}$ знак минус исчезает, и в результате формула (1) приобретает вид

$$
\int_{\left(l_{3}\right)_{\min }}^{\left(l_{3}\right)_{\max }} \frac{e^{\lambda S\left(l_{1}, l_{2}, l_{3}\right)}}{S\left(l_{1}, l_{2}, l_{3}\right)} \frac{e^{\lambda S\left(l_{3}, l_{4}, l_{5}\right)}}{S\left(l_{3}, l_{4}, l_{5}\right)} l_{3} d l_{3}=\int_{\left(l_{6}\right)_{\min }}^{\left(l_{6}\right)_{\max }} \frac{e^{\lambda S\left(l_{1}, l_{5}, l_{6}\right)}}{S\left(l_{1}, l_{5}, l_{6}\right)} \frac{e^{\lambda S\left(l_{2}, l_{4}, l_{6}\right)}}{S\left(l_{2}, l_{4}, l_{6}\right)} l_{6} d l_{6} .
$$




\section{4. ГЕОМЕТРИЧЕСКАЯ ДУАЛЬНОСТЬ ДЛЯ УГЛОВЫХ КОЭФФИЦИЕНТОВ - ОБОБЩЕНИЕ АМПЛИТУДЫ ВЕНЕЦИАНО}

Схема рис. 4 подсказывает, что имеется и еше один выбор преобразования, удовлетворяющего ФУП. Для данного ребра возьмем в качестве квантового числа его угловой коэффициент $k$ (если концы ребра в фиксированной системе координат, не обязательно ортогональной, суть $\left(x_{1}, y_{1}\right)$ и $\left(x_{2}, y_{2}\right)$, то $\left.k=\left(y_{2}-y_{1}\right) /\left(x_{2}-x_{1}\right)\right)$. Нетрудно видеть, что если величины $k_{1}, k_{2}, k_{3}, k_{4}$ и $k_{5}$ на рис. 6 заданы, то $k_{6}$ определяется однозначно. Вьпишем формулу для нахождения $k_{6}$ в следуюшем виде:

$$
\frac{k_{3}-k_{2}}{k_{3}-k_{1}} \cdot \frac{k_{3}-k_{5}}{k_{3}-k_{4}}=\frac{k_{6}-k_{2}}{k_{6}-k_{4}} \cdot \frac{k_{6}-k_{5}}{k_{6}-k_{1}} .
$$

Это соотношение имеет форму

$$
F(A B D) \cdot G(B C D)=H(A B C) \cdot K(A C D) .
$$

Под этим мы подразумеваем, что левая часть является произведением двух выражений, соответствующих треугольникам $A B D$ и $B C D$, тогда как правая часть соответствует аналогично треугольникам $A B C$ и $A C D$. Поэтому соотношение (8) аналогично (3), хотя оно "мультипликативно", а не "аддитивно".

Отметим, что, во-первых, уравнение (8) имеет также решение $k_{6} \equiv k_{3}$, которое нас не интересует. Будем считать, что мы его отбросили. Во-вторых, преобразование $f: k_{3} \mapsto$ $k_{6}$ при данных $k_{1}, k_{2}, k_{4}$ и $k_{5}$ инволютивно.

То, что преобразование $f$ удовлетворяет уравнению пентагона, следует из того, что пятиугольник, в котором заданы угловые коэффициенты всех сторон и двух диагоналей, определен однозначно с точностью до подобия и сдвига.

Очень важно, что существуют еще два мультипликативных соотношения, дающих ту же зависимость $f: k_{3} \mapsto k_{6}$ и имеюших ту же форму (9):

$$
\frac{k_{1}-k_{2}}{k_{1}-k_{3}} \cdot \frac{k_{4}-k_{3}}{k_{4}-k_{5}}=\frac{k_{4}-k_{2}}{k_{4}-k_{6}} \cdot \frac{k_{1}-k_{6}}{k_{1}-k_{5}}
$$

И

$$
\frac{k_{2}-k_{1}}{k_{2}-k_{3}} \cdot \frac{k_{5}-k_{3}}{k_{5}-k_{4}}=\frac{k_{2}-k_{6}}{k_{2}-k_{4}} \cdot \frac{k_{5}-k_{1}}{k_{5}-k_{6}} .
$$

В качестве аналога формулы (5) можно взять

$$
\frac{d k_{3}}{\left(k_{3}-k_{2}\right)\left(k_{3}-k_{5}\right)}=-\frac{d k_{6}}{\left(k_{6}-k_{2}\right)\left(k_{6}-k_{5}\right)} .
$$

Несложно проверить, что (12) следует из формулы (8) при условии $k_{6} \not \equiv k_{3}$.

Важной особенностью формул (8)-(12) является то, что они остаются верными после мёбиусова (рационального) преобразования всех $k$ :

$$
k_{j} \mapsto \frac{a k_{j}+b}{c k_{j}+d}, \quad j=1, \ldots, 6 .
$$


Такие преобразования соответствуют просто другому выбору системы координат для диаграммы рис. 6 .

Наиболее обшая формула типа (1), которую мы можем вывести из (8)-(12), получается, если возвести все члены в (8) в некоторую степень $\alpha$, в (10) - в некоторую степень $\beta$, в (11) - в некоторую степень $\gamma$ и перемножить соответствующие члены этих уравнений и уравнения (12). Используя соотношения

$$
k_{3}=k_{2} \Leftrightarrow k_{6}=k_{5} \quad \text { и } \quad k_{3}=k_{5} \Leftrightarrow k_{6}=k_{2},
$$

мы можем выбрать, например, кривую, соединяюшую $k_{2}$ и $k_{5}$, как путь интегрирования и записать окончательную формулу в виде

$$
\begin{aligned}
& \int_{k_{2}}^{k_{5}} A\left(k_{3}, k_{1}, k_{2} \mid-\alpha, \beta,-\gamma\right) A\left(k_{3}, k_{4}, k_{5} \mid-\alpha,-\beta, \gamma\right) d k_{3}= \\
& \quad=\int_{k_{2}}^{k_{5}} A\left(k_{6}, k_{4}, k_{2} \mid-\alpha, \beta, \gamma\right) A\left(k_{6}, k_{1}, k_{5} \mid-\alpha,-\beta,-\gamma\right) d k_{6},
\end{aligned}
$$

где

$$
A(l, m, n \mid \lambda, \mu, \nu)=(l-m)^{\lambda-\mu}(m-n)^{\mu-\nu}(n-l)^{\nu-\lambda-1} .
$$

Обе части формулы (13) обобшают известное выражение Венециано $[10,11]$ для четырехточечной амплитуды. Чтобы это увидеть, положим

$$
k_{1}=k_{4}=\infty, \quad k_{2}=0, \quad k_{5}=1 .
$$

Тогда, например, левая часть (13) дает с точностью до постоянного множителя

$$
\int_{0}^{1} k_{3}^{\alpha-\gamma-1}\left(k_{3}-1\right)^{\alpha+\gamma-1} d k_{3}
$$

что совпадает с амплитудой Венециано с точностью до очевидного изменения обозначений.

Будет несомненно очень интересно сравнить пятиточечную и вообще $N$-точечную амплитуды, которые можно получить таким образом, с амплитудами из классических работ [12-15].

\section{5. ОБСУЖДЕНИЕ: ТЕОРЕТИКО-ГРУППОВЫЕ ЗАМЕЧАНИЯ}

Объясним, почему мы считаем, что наши конструкции связаны с группами Ли. Рассмотрим два вида преобразований, действуюших на точки $(x, y)$ евклидовой плоскости: сдвиг на $a$ вдоль оси $x-$

$$
S(a):(x, y) \mapsto(x+a, y)
$$

и поворот на угол $\phi-$

$$
R(\phi):(x, y) \mapsto(x \cos \phi-y \sin \phi, x \sin \phi+y \cos \phi) .
$$


Сушествование треугольника со сторонами $l, m, n$ и внешними углами $\alpha, \beta, \gamma$ означает равенство

$$
R(\alpha) \circ S(n) \circ R(\beta) \circ S(l) \circ R(\gamma) \circ S(m)=\mathbf{1}
$$

Отметим, что группа движений евклидовой плоскости трехпараметрическая, и поэтому $\alpha, \beta$ и $\gamma$ могут быть определены по известным $l, m$ и $n$. Соотношения, аналогичные (16), могут быть выписаны также для четырех- и пятиугольников, и все геометрические построения раздела 3 могут быть описаны в терминах таких соотношений.

Конструкции же раздела 4 никак не связаны с евклидовым расстоянием, так что, по нашему мнению, здесь более подходяшей является трехпараметрическая группа, порожденная преобразованием $S(a)(15)$ и преобразованием

$$
T(\kappa):(x, y) \mapsto(x, y+\kappa x)
$$

Тогда сушествование треугольника, проекции сторон которого на ось $x$ суть $a, b, c$ (где $a+b+c=0)$, а их угловые коэффициенты $-l, m, n$, описывается равенством

$$
S(a) \circ T(l-m) \circ S(b) \circ T(m-n) \circ S(c) \circ T(n-l)=\mathbf{1} .
$$

С алгебраической точки зрения, группа, порожденная преобразованиями $S(a)$ и $T(\kappa)$, есть не что иное как группа Гейзенберга. Это сразу видно из соотношения

$$
T(\kappa) \circ S(a) \circ T(-\kappa) \circ S(-a)=C(\kappa a),
$$

где

$$
C(a):(x, y) \mapsto(x, y+a)
$$

- центральный элемент для любого $a$.

Мы считаем, что изучение соотношений типа $(16),(17)$ в бо́льших группах является алгебраическим ключом для построения широких обобщений теории струн.

Благодарности. Один из авторов (И.Г.К.) с удовольствием благодарит Токийский городской университет (Tokyo Metropolitan University) за грант на проведение исследований (TMU Fellowship for research), профессора этого университета С. Сайто за гостеприимство, а также Российский фонд фундаментальных исследований за поддержку по гранту 98-01-00895. 


\section{Список литературы}

[1] S. Saito. Phys. Rev. Lett. 1987. V. 59. № 16. P. 1798.

[2] S. Saito. Integrability of strings. In: Nonlinear Fields: Classical, Random, Semiclassical. Eds. P. Garbaczewski and Z. Popovicz. Singapore: World Scientific, 1991. P. 286.

[3] K. Sogo. J. Phys. Soc. Japan. 1987. V. 56. № 7. P. 2291.

[4] S. Saito. J. Phys. A. 1997. V. 30. P. 8289.

[5] A. B. Zamolodchikov. Commun. Math. Phys. 1981. V. 79. P. 489.

[6] R. M. Kashaev. On discrete 3-dimensional equations associated with the local Yang-Baxter relation. Preprint ENSLAPP-L-569/95. Lyon: ENS-Lyon, 1995; solv-int/9512005.

[7] Р. М. Кашаев, И.Г. Корепанов, С. М. Сергеев. ТМФ. 1998. Т. 117. № 3. С. 370; R.M. Kashaev, I. G. Korepanov, and S.M. Sergeev. Functional tetrahedron equation, solv-int $/ 9801015 ;$ В. В. Фок, Л. О. Чехов. Квантовые модулярные преобразования, соотношение пятиугольника и геодезические. Тр. МИАН (принято в печать).

[8] R. M. Kashaev, S. M. Sergeev. Commun. Math. Phys. 1998. V. 195. P. 309; On pentagon, ten-term, and tetrahedron relations, q-alg/9607032.

[9] Р. М. Kauaes. ТМФ. 1999. Т. 118. № 3. C. 398.

[10] G. Veneziano. Nuovo. Cimento A. 1968. V. 57. P. 190.

[11] M. B. Green, J.H. Schwarz, E. Witten. Superstring Theory. V. 1. Cambridge: Cambridge Univ. Press, 1988.

[12] K. Bardakçi, H. Ruegg. Phys. Lett. B. 1968. V. 28. № 5. P. 342.

[13] M. A. Virasoro. Phys. Rev. Lett. 1969. V. 22. № 1. P. 37.

[14] C. J. Goebel, B. Sakita. Phys. Rev. Lett. 1969. V. 22. № 6. P. 257.

[15] Z. Koba, H. B. Nielsen. Nucl. Phys. B. 1969. V. 12. P. 517.

Поступила в редакцию 18.XII.1998 г. 